\title{
Percepción de bienestar en experiencias inclusivas de blended learning
}

\section{Wellbeing Perception in Inclusive Experiences of Blended Learning}

\author{
Alba María Hernández-Sánchez ${ }^{1}$ \\ albamaria@ugr.es \\ José Antonio Ortega Carrillo \\ jaorte@ugr.es \\ Universidad de Granada, España
}

\section{Resumen:}

Este artículo analiza el grado de bienestar del alumnado participante en un programa formativo desarrollado en la modalidad de blended learning en el que participan personas con y sin discapacidad auditiva. La pertinencia de esta temática queda justificada por la escasa presencia en la literatura científica de investigaciones sobre educación inclusiva en entornos virtuales.

El estudio exploratorio muestra datos parciales extraídos de un cuestionario de elaboración propia, validado en experiencias anteriores. Los resultados ofrecidos relacionan el grado de cumplimiento de las expectativas del alumnado y la sensación de bienestar percibida durante el desarrollo de la acción formativa, en el contexto inclusivo.

Las conclusiones apuntan a la consecución generalizada de un alto grado de bienestar estimado desde el análisis de evidencias referidas al acompañamiento afectivo y a la personalización de las estrategias, recursos y dinámicas tecno-

\begin{abstract}
:
This study analyses the level of well-being of the deaf and non-deaf students that participate in a blended learning experience. This study finds its relevance in the lack of scientific literature regarding inclusive education in virtual environments.

The exploratory study shows a set of partial data of an ad hoc survey, validated in previous experiences. The results relate the students' expectations and the perception of well-being along the development of the experience, in an inclusive context. The findings indicate that there is a widespread high level of well-being which has been inferred from the analysis of evidence related to affective assistance and the personalization of technological and didactic strategies, resources and dynamics. The indicators analysed highlight accessibility, the increase of creative and collaborative participation, the face-to-face and virtual well-being and the avoidance of anxiety, frustration and reluctance.
\end{abstract}

1 Dirección para correspondencia (correspondence address):

Alba María Hernández Sánchez. Dpto. de Didáctica y Organización Escolar. Facultad de Ciencias de la Educación. Universidad de Granada. Campus Universitario de Cartuja. 18071 Granada (España). 
lógico-didácticas según las preferencias individuales.

Los indicadores analizados señalan la observancia de la accesibilidad, el fomento de la participación creativa y colaborativa, el disfrute en la convivencia presencial y en línea y la evitación de situaciones de ansiedad, frustración y desgana.

\section{Palabras clave:}

Blended learning; educación inclusiva; bienestar; afectividad; discapacidad.

\section{Keywords:}

Blended learning; inclusive education; well-being; affectivity; disability.

\section{Résumé:}

Cet article analyse le degré de bien-être des étudiants qui ont participé dans un programme de formation, développé avec la modalité blended learning auquel participent des personnes avec et sans handicap auditif. La pertinence de cette thématique est justifiée par la faible présence de littérature scientifique de recherches sur une éducation inclusive dans des environnements virtuels.

L'étude exploratoire montre des données partielles extraites d'un questionnaire validé lors d'expériences précédentes. Les résultats proposés font le lien entre le degré d'accomplissement des attentes des élèves et la sensation de bien-être perçue pendant le développement de l'action formative, dans le contexte inclusif.

Les conclusions montrent l'obtention généralisée d'un haut degré de bien-être estimé à partir de l'analyse d'évidences liées à l'accompagnement affectif et à la personnalisation des stratégies, des ressources et des dynamiques technologiques - didactiques selon les préférences individuelles.

Les indicateurs analysés marquent l'observance de l'accessibilité, la promotion de la participation créative et collaborative, le plaisir dans la cohabitation face-à-face et en ligne, en plus de la prévention de situations d'anxiété, de frustration et de découragement.

\section{Mots clés:}

Blended learning; éducation inclusive; bien-être; affectivité; handicap.

Fecha de recepción: 12-12-2015

Fecha de aceptación: 28-4-2016

\section{Introducción}

La educación semipresencial o blended learning se viene concibiendo como una modalidad de aprendizaje con valor añadido que conjuga experiencias de construcción de conocimiento de forma presencial y en línea. Tal combinación, como señalan Garrison \& Vaughan (2008), implica la transformación de la organización de la enseñanza y del aprendizaje sin alterar los valores fundamentales de la educación superior.

Autores como Collopy \& Marshall (2009) y Garrison (2011) han subrayado que la modalidad de blended learning es una opción que aporta 
respuestas válidas a las necesidades de innovar la praxis educativa al proponer cambios paradigmáticos coherentes con el desarrollo de la sociedad digital; invitando a las instituciones educativas a 'convertirse en poderosos escenarios de aprendizaje, donde los estudiantes investigan, comparten, aplican y reflexionan' (Pérez, 2012, p.70).

Todos los participantes involucrados en el desarrollo de la acción formativa tienen la posibilidad de combinar y crear múltiples estrategias, actividades y recursos que según Llorente \& Cabero (2009, p.94) posibilitan una experiencia más efectiva donde 'los estudiantes aprenden más y disfrutan más que si lo hacen exclusivamente con la enseñanza online o presencial'.

Las características propias del blended learning han de focalizarse hacia la consecución del ideal de educación inclusiva para hacer realidad los compromisos internacionales plasmados en la Convención sobre los Derechos de las Personas con Discapacidad promovida por la UNESCO en 2006, ya que permiten, como subrayan Cabero, Llorente \& Puentes (2010), la flexibilización espacio-temporal, la mayor accesibilidad a los materiales, la interacción comunicativo-digital y el refuerzo de la pertenencia al grupo. El ideal de inclusión educativa demanda crear 'procesos abiertos y flexibles, capaces de amoldarse a las nuevas realidades diversas; procesos pensados, consensuados y llevados a cabo desde el propio centro educativo' (Martínez \& Gómez, 2013, p.17), donde es sumamente importante que se apueste por modalidades como la del blended learning que, lejos de asumir estándares descontextualizados, diseñan actividades de aprendizaje congruentes con los objetivos educativos (Garrison, 2011).

El diseño, desarrollo y evaluación de una acción formativa semipresencial fundamentada en los principios de la educación inclusiva implica la comprensión y materialización de las siguientes premisas (Ainscow, 2009): la inclusión es un proceso que busca de forma continua la mejora para responder a la diversidad; preocupada por la identificación y eliminación de barreras que impidan la presencia, la participación y el logro de todo el alumnado; poniendo especial énfasis en aquellos grupos de estudiantado que podrían estar en riesgo de marginalización, exclusión o bajo rendimiento.

Estas premisas han de concretarse en un contexto renovado desde la conformación de una cultura, una política y unas prácticas inclusivas (Booth \& Ainscow, 2011), orientadas a la superación de una nueva 
forma de desigualdad, la denominada brecha digital o e-exclusión (Marín, Gutiérrez \& Aguaded, 2014). Incidiéndose en la aplicación de los principios del Diseño Universal que tal como exponen Sala, Sánchez, Giné \& Díaz (2014, p.149) persiguen 'romper las barreras de aprendizaje presentes en los procesos de enseñanza para que todos los estudiantes, sean cuales sean sus particularidades, puedan acceder en igualdad de oportunidades'. Tal y como se observa, la problemática de investigación que se aborda redunda en la superación de un modelo de enseñanza en línea que excluye de forma directa o indirecta a ciertas personas o colectivos de personas, haciendo a menudo que el inmovilismo hacia prácticas, políticas y culturas inclusivas repercuta en la insatisfacción del alumnado.

Tal formalización ha de permitir que todo el alumnado tenga la posibilidad de desarrollar en plenitud sus capacidades, desde diseños y desarrollos de acciones formativas coherentes con las demandas de la sociedad de la información y la comunicación, que solicitan que el alumnado sea capaz de:

adaptarse a un ambiente que se modifica rápidamente; saber trabajar en equipo; aplicar propuestas creativas y originales para resolver problemas; la capacidad para aprender; desaprender y reaprender; saber tomar decisiones y ser independiente; aplicar las técnicas del pensamiento abstracto; y saber identificar problemas y desarrollar soluciones (Llorente, Cabero \& Barroso, 2015, p.230).

En este contexto innovador propugnamos un blended learning inclusivo que, contemplado desde los principios de la psicología positiva, permita a todos los integrantes de la comunidad ciber-formativa vivenciar un estado óptimo de experiencia interna que pueda conseguirse 'cuando la energía psíquica (o atención) se utiliza para obtener unas metas realistas y cuando las habilidades encajan con las oportunidades para actuar' (Csikszentmihalyi, 2013, p.19). Ello ha de permitir alcanzar un estado de flujo (flow) en los actores involucrados que incida en la percepción de bienestar dentro de la comunidad de aprendizaje. Además de la participación activa y comprometida de cada persona, el planteamiento y desarrollo de tareas que se puedan realizar (Carr, 2007), favorecerá la declaración de bienestar que se percibe cuando el reto propuesto está en equilibrio con las habilidades de la persona. 


\section{Objetivo de la investigación}

El presente trabajo forma parte de un proceso de investigación en la acción centrado en la mejora continua de las actuaciones educativas que se desarrollan, la capacitación para el cambio y la innovación y la generación de conocimiento social práctico emancipatorio (Colás, Buendía \& Hernández, 2009). El diseño investigativo se orienta hacia la transformación de la realidad (Durán \& Giné, 2011) desde una perspectiva holística que incluye a todos los agentes y procesos involucrados.

Los resultados de investigación compilados en este artículo pretenden describir y valorar el grado de bienestar producido por el desarrollo de un programa educativo inclusivo aplicado en la modalidad de blended learning. Por consiguiente se pretende relacionar el bienestar declarado con aspectos tales como el cumplimiento de las expectativas del alumnado, la accesibilidad, la participación, el disfrute, el avance en la construcción del conocimiento y la evitación de situaciones adversas, en un contexto de educación inclusiva en entornos presenciales y virtuales.

En concreto, se podría decir que en relación al objetivo general, se pretende:

- Analizar las motivaciones y dificultades que inciden en la participación del alumnado.

- Conocer el grado de cumplimiento de las expectativas del alumnado en relación a la experiencia formativa.

- Determinar la influencia del equipo tutorial, los/as compañeros/as, la coordinación, el material y la propia perseverancia e implicación del alumnado en el aprendizaje percibido.

- Explicar la sensación de bienestar percibida por el alumnado desde las estrategias y dinámicas propias del modelo de eLearning inclusivo.

\section{Método de investigación}

\subsection{Contextualización}

Las informaciones generadas en este trabajo provienen de una acción educativa innovadora desarrollada en la modalidad de blended learning en la que interactúan personas con y sin discapacidad auditiva. En con- 
creto, el estudio se focaliza en torno a la $3 .^{a}$ Edición del diploma de Lengua de Signos Española y su Interpretación aplicada a la enseñanza presencial y virtual (15/D/005) que organiza la Escuela Internacional de Posgrado de la Universidad de Granada. La creación de un entorno y unas experiencias propicias para impulsar las directrices sobre política y educación inclusiva de la UNESCO (2009), se materializa con el desarrollo de 'cambios y modificaciones de contenidos, enfoques, estructuras y estrategias' (Echeita \& Ainscow, 2011, p.1) que garantizan una mayor y mejor participación de todo el alumnado.

La incorporación de medidas, dinámicas y estrategias que favorecen la generación de una educación inclusiva se han desarrollado y estudiado a través de dos dimensiones: la cultura y la práctica inclusiva. Pensamos con Booth \& Ainscow (2011) que la creación de culturas inclusivas supone la generación de espacios colaborativos, acogedores y estimulantes donde todo el alumnado se sienta valorado. Del mismo modo que el desarrollo de prácticas inclusivas se centra en el aseguramiento de actividades que motiven la participación del conjunto del alumnado, propiciando apoyos y dinámicas que permitan superar las posibles barreras de aprendizaje y la movilización y orquestación de recursos.

La acción educativa se desarrolla como un título propio de posgrado planeado según las orientaciones y planteamientos que expone el Marco Común Europeo de Referencia para las Lenguas (MCERL). Se ha desarrollado a lo largo de los meses comprendidos entre febrero y junio de 2014 (ambos inclusive) en un Campus Virtual Accesible e Inclusivo de generación propia. La institución que acoge la acción formativa en cuestión ha mostrado, a través de una evaluación externa del diploma (del 66,7 de índice de respuesta), que "la acción formativa ha cumplido las expectativas del alumnado ya que el $92 \%$ la valora globalmente como "Muy bien" o "Excelente", obteniéndose una media de 4,58 sobre 5 , y el $96 \%$ la recomendaría.

\subsection{Instrumento}

Se elaboró un cuestionario general ad hoc para la evaluación de la calidad y la satisfacción. (Versión II) que incluye una nueva dimensión en relación con la versión primera, dirigida a analizar el grado de bienestar percibido a través del modelo de eLearning inclusivo. En este trabajo se presentan resultados de los datos generales de la población del estudio 
y de las dimensiones: a) expectativas, b) evaluación y motivación de los aprendizajes y c) grado de bienestar desde el enfoque inclusivo. Los ítems incluidos en el estudio usaron dos tipos de formato de respuesta, ambas cuantificables: de respuesta dicotómica y de escala tipo Likert con 4 grados de elección (en la última dimensión se añadió la opción de no sabe/no contesta - ns/nc).

Los apartados referidos a las expectativas, la evaluación y la motivación en el programa formativo aglutinan un total de 31 ítems, orientados al análisis de los procesos vivenciados por el alumnado, tanto a nivel de intereses y cumplimiento de los mismos, como a nivel de consecución de aprendizajes y satisfacción del proceso.

Por su parte, el apartado final del cuestionario referido al estudio del grado de bienestar del alumnado, está compuesto por 38 ítems que se agrupan en torno a las cuatro subdimensiones que componen las dos dimensiones principales del Index de Educación Inclusiva de Booth \& Ainscow (2011): la construcción de una comunidad y el establecimiento de valores inclusivos conforma la dimensión de cultura inclusiva; y, la movilización de los recursos y la organización de los aprendizajes constituyen la dimensión de prácticas inclusivas.

Para vertebrar la argumentación de los resultados de los ítems se ha realizado un agrupamiento por dimensiones que se recoge en la tabla 1, (la dimensión c se ha subdividido en las subdimensiones correspondientes):

Tabla 1. Dimensiones y agrupamiento de ítems analizadas en la investigación.

\begin{tabular}{|c|c|c|}
\hline \multicolumn{2}{|l|}{ Dimensión } & Ítems \\
\hline \multicolumn{2}{|c|}{ Expectativas } & $\begin{array}{l}23 \text { ítems }(8.1,8.2,8.3,8.4,8.5,9,10.1,10.2,11, \\
12,13.1,13.2,13.3 ., 13.4,13.5,13.6,13.7,13.8, \\
13.9,13.10,13.11,13.12,13.13) .\end{array}$ \\
\hline \multicolumn{2}{|c|}{ Evaluación y motivación } & 8 ítems $(21.1,21.2,21.3,21.4,21.5,21.6,24,25)$. \\
\hline \multicolumn{2}{|c|}{$\begin{array}{l}\text { Grado de bienestar des- } \\
\text { de el enfoque inclusivo }\end{array}$} & 38 ítems (del 26.1 al 26.38) \\
\hline \multirow{2}{*}{$\begin{array}{l}\text { c. } 1 . \\
\text { Cultura } \\
\text { inclusiva }\end{array}$} & $\begin{array}{l}\text { Construir } \\
\text { comunidad }\end{array}$ & $\begin{array}{l}7 \text { ítems }(26.14,26.15,26.23,26.27,26.28,26.29 \text {, } \\
26.30) \text {. }\end{array}$ \\
\hline & $\begin{array}{l}\text { Valores } \\
\text { inclusivos }\end{array}$ & $\begin{array}{l}10 \text { ítems }(26.5,26.8,26.10,26.17,26.18,26.19 \\
26.20,26.31,26.32,26.37) .\end{array}$ \\
\hline \multirow{2}{*}{$\begin{array}{l}\text { c. } 2 \text {. } \\
\text { Prácticas } \\
\text { inclusivas }\end{array}$} & $\begin{array}{l}\text { Organización } \\
\text { aprendizaje }\end{array}$ & $\begin{array}{l}13 \text { ítems }(26.1,26.2,26.3,26.4,26.6,26.7,26.9, \\
26.11,26.12,26.22,26.33,26.34,26.36) .\end{array}$ \\
\hline & $\begin{array}{l}\text { Movilización } \\
\text { de recursos }\end{array}$ & $\begin{array}{l}8 \text { ítems }(26.13,26.16,26.21,26.24,26.25,26.26 \text {, } \\
26.35,26.38) .\end{array}$ \\
\hline
\end{tabular}


El cuestionario fue aplicado en un estudio piloto anterior y validado a nivel de contenido por criterio de jueces. El coeficiente de correlación intraclase muestra un nivel de acuerdo muy alto $(0,981)$ entre los nueve evaluadores. Igualmente, se ha medido la consistencia interna de los ítems del instrumento a través del coeficiente Alfa de Cronbach, obteniéndose una fiabilidad muy alta, de 0,988.

\subsection{Población y perfil de la muestra}

La muestra está formada por el 92,31 \% de la población total, quedando configurada por 36 de 39 inscritos en el curso. La mayoría de los participantes son mujeres $(83,3 \%)$ oyentes $(91,7 \%)$, siendo parte del grupo de alumnado dos personas sordas y una con hipoacusia. Por su parte, la mayor parte de la muestra se sitúa entre los 20 y los 25 años (63,9\%), mientras que el resto de las edades se distribuyen entre los 18 y los 65, siendo el segundo núcleo de mayor densidad poblacional el comprendido entre los 26 y los 35 años (19,4\%).

Algo menos de la cuarta parte $(22,2 \%)$ posee estudios universitarios, mientras que el resto son alumnos finalistas de grado de diversas universidades, si bien uno de los matriculados, no cumpliendo tal condición se ha acogido a la posibilidad de acceder al curso de forma extraordinaria al acreditar su relación profesional con la temática del citado diploma, según lo previsto en la normativa aprobada por el Consejo de Gobierno de la Universidad en cuestión en 2013. Tal participante tiene hipoacusia por lo que la aplicación de la citada norma ha favorecido su discriminación positiva.

El 83,33\% manifiesta no disponer de conocimientos previos acerca de la temática del diploma y solo seis personas dicen conocer la lengua de signos española: dos de ellas por ser su lengua materna y otras dos personas manifiestan el nivel de usuario competente, adquirido como segunda o tercera lengua. Un quinto caso presenta un dominio de usuario "independiente" equivalente a un nivel B2 o avanzando, mientras que el sexto caso dice tener conocimientos como usuario "básico" (nivel A1-A2).

Preguntados sobre la experiencia anterior como alumno de formación en línea, un 91,7 \% declara no tener ninguna experiencia y un $94,4 \%$ dice no hacer sido profesor de cursos de enseñanza a distancia en entornos virtuales. 


\section{Resultados}

\subsection{Participación: razones y dificultades}

Las argumentaciones sobre los motivos para participar en esta propuesta formativa se recogen en la figura 1:

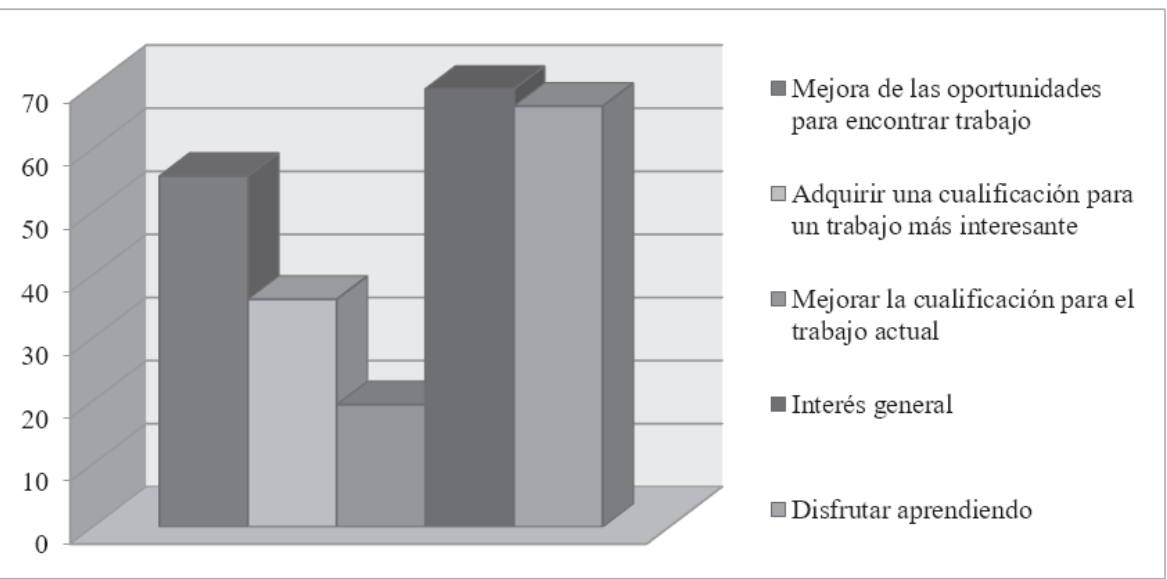

Figura 1. Porcentaje atribuido a las razones para la participación.

Las dos razones que más sobresalen coinciden con el interés general sobre la temática $(69,4 \%)$ y sobre la posibilidad de disfrutar aprendiendo a través de la experiencia formativa $(66,7 \%)$. Es igualmente destacable que la mitad de los participantes $(55,6 \%)$ forme parte del curso creyendo que le puede ayudar a mejorar sus oportunidades de encontrar trabajo.

La figura 2 recoge información sobre dificultades u obstáculos detectados en el desarrollo del diploma. Analizadas las dificultades encontradas, se apunta a la falta de tiempo (50\%), al exceso de trabajo $(22,2 \%)$, a la ausencia de experiencia en entornos virtuales $(19,4 \%$ y a algunas dificultades técnicas $(11,1 \%)$.

El resto de indicadores no presentan relevancia estadística, lo que indica que no han existido otras dificultades distintas a las anteriores. Así, ni el desinterés por el tema ni la inscripción obligada en el diploma se configuran como condicionantes negativos. Tampoco lo han sido la desmotivación, la soledad, la carencia de apoyo tutorial y la falta de medios y materiales, panorama que resalta la calidad conseguida por la acción formativa. 


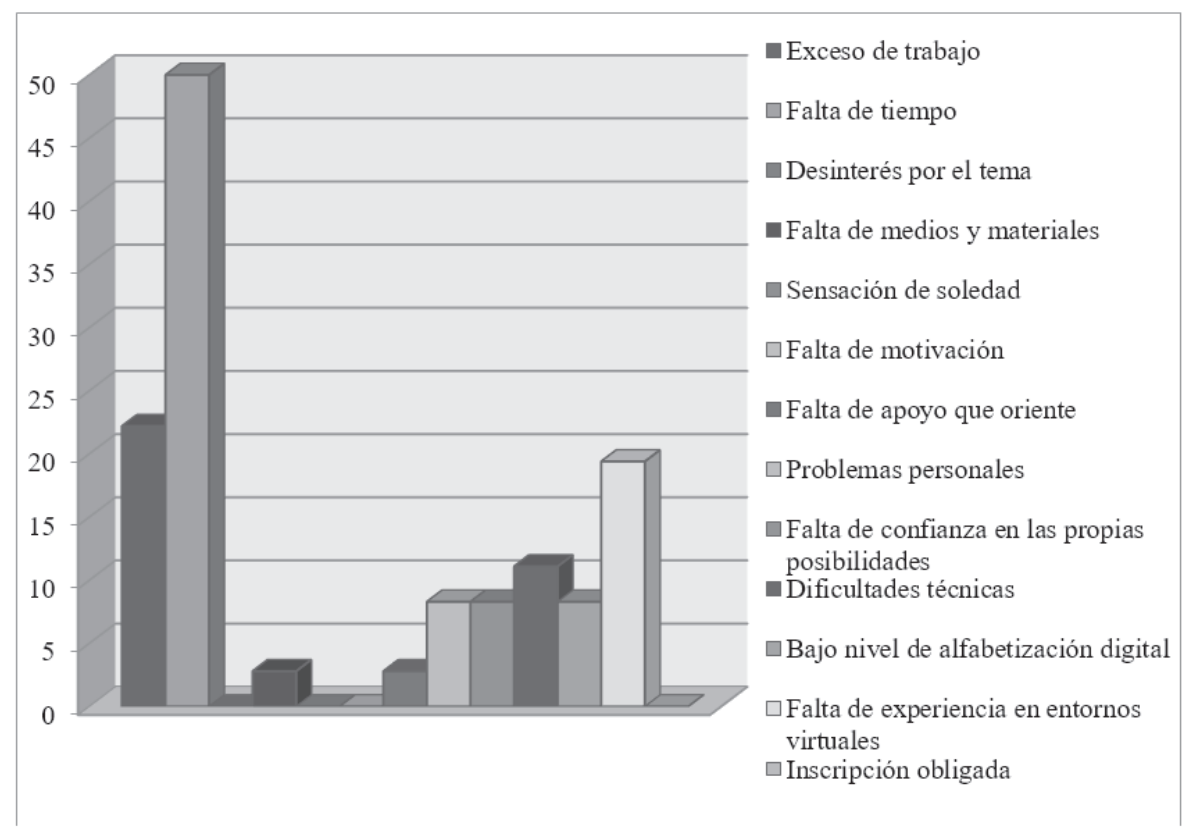

Figura 2. Circunstancias que pudieron influir negativamente en el proceso de aprendizaje.

\subsection{Hacia la experiencia óptima: alto cumplimiento de expectativas}

Preguntados sobre el grado de cumplimiento de las expectativas puestas en el programa formativo, ocho de cada diez $(80,6 \%)$ declaran muy alto grado de cumplimiento y 2 de cada $10(19,4) \%$ expresa haber cumplido bastante tales expectativas. En la misma línea, los participantes lo consideran de interés (un 83,3\% muy interesante y un 19,4\% bastante interesante) y agradable (muy agradable el $66,7 \%$ y bastante el $33,3 \%$ ), obteniendo un nivel de satisfacción y una sensación de progreso muy altas.

Valorado el programa en función de los aprendizajes conseguidos, el $80,6 \%$ lo valora como muy positivo y el 16,7 \% como bastante positivo; únicamente un participante que no obtuvo calificación positiva, reconoció haber aprendido poco. Estos datos se refuerzan con la estimación de "si se progresó en el estudio tal como se esperaba" ya que el 50 \% lo hizo con plena satisfacción y el 47,2\% con bastante satisfacción.

Igualmente, la mayor parte del alumnado indica que se ha sentido protagonista de su proceso de aprendizaje y que ha tenido la sensación 
de ir alcanzando las metas que se marcaban (con un $97,2 \%$ y un 94,4 $\%$, respectivamente).

\subsection{Factores que han incidido en el aprendizaje}

La figura 3 incluye los resultados desglosados de los distintos factores que han condicionado los aprendizajes.

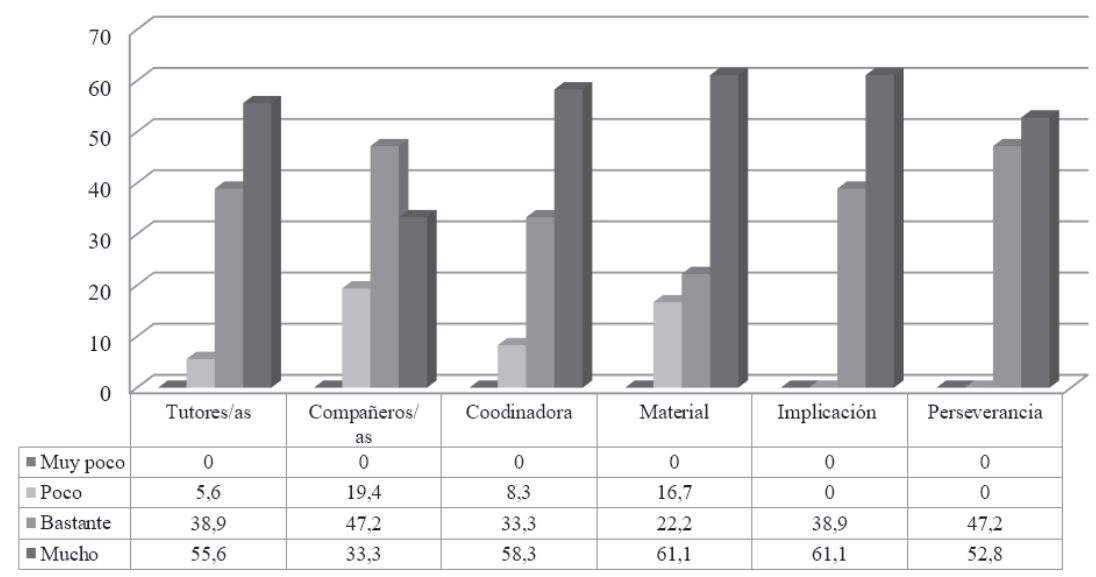

Figura 3. Porcetajes de influencia de los factores que condicionan el aprendizaje.

La valoración del factor implicación indica el grado de compromiso en el desarrollo de la acción formativa, siendo muy buena, ya que un 61,1 $\%$ declara una implicación muy alta y alta para el restante 38,9\%. En lo referido a la cota de perseverancia declarada es igualmente muy significativa ( $52,8 \%$ muy alta y $47,2 \%$ bastante alta).

Al preguntarse sobre la influencia de ciertos elementos didácticos en el buen desarrollo de los procesos de aprendizaje, se observa que es el acompañamiento tutorial el factor mejor valorado con un $94,5 \%$, seguido de la acción de la coordinadora con un 91,6\%. Igualmente resultan bien valorados la interacción entre iguales $(80,5 \%$ ) y la calidad de los materiales hipermedia ofrecidos en el campus virtual estimada favorablemente por un $83,3 \%$.

Estas estimaciones sugieren la existencia de una alta motivación y de un 
buen nivel de ejecución generalizado, agentes generadores de un clima muy favorable para la construcción de los aprendizajes en línea y presenciales.

\subsection{La importancia del bienestar en una experiencia educativa inclusiva}

La sensación de bienestar percibida desde las estrategias y dinámicas propias del modelo de eLearning inclusivo, analizada por el último bloque de 38 ítems del cuestionario, es considerada en su conjunto por 8 de cada 10 alumnos como alta y muy alta. Se realiza un análisis selectivo de esta dimensión en virtud de las características propias de este trabajo, agrupando los comentarios en función a los dos ámbitos ya explicitados: los indicadores de cultura inclusiva y los aspectos referentes a la práctica tecnológico-didáctica inclusiva. La tabla 2 muestra los porcentajes de respuesta de este conjunto de indicadores:

Tabla 2. Desglose de resultados de los ítems referentes a la dimensión de grado de bienestar declarado desde el enfoque de educación inclusiva.

\begin{tabular}{|c|c|c|c|c|c|}
\hline Ítem/ Porcentajes & $\begin{array}{l}\text { Mu- } \\
\text { cho }\end{array}$ & $\begin{array}{l}\text { Bas- } \\
\text { tante }\end{array}$ & Poco & $\begin{array}{l}\text { Muy } \\
\text { poco }\end{array}$ & $\mathrm{Ns} / \mathrm{Nc}$ \\
\hline 1. La flexibilidad ofrecida en ciertos plazos & 75 & 22,2 & 2,8 & 0 & 0 \\
\hline $\begin{array}{l}\text { 2. El hecho de que se hayan tenido en cuenta ciertas } \\
\text { eventualidades y dificultades personales }\end{array}$ & 75 & 13,9 & 2,8 & 0 & 8,3 \\
\hline $\begin{array}{l}\text { 3. La colaboración del alumnado en la co-evaluación } \\
\text { realizada al comentar tareas desde espacios colaborati- } \\
\text { vos (espacios de foro) }\end{array}$ & 63,9 & 25 & 2,8 & 2,8 & 5,6 \\
\hline $\begin{array}{l}\text { 4. La claridad de criterios de evaluación expresada en las } \\
\text { e-rúbricas }\end{array}$ & 55,6 & 22,2 & 8,3 & 0 & 13,9 \\
\hline 5. El acompañamiento afectivo del equipo tutorial & 72,2 & 22,2 & 2,8 & 0 & 2,8 \\
\hline 6. La celebración de videoconferencias grupales & 66,7 & 25 & 5,6 & 0 & 2,8 \\
\hline 7. La celebración de videoconferencias individuales & 63,9 & 25 & 5,6 & 0 & 5,6 \\
\hline $\begin{array}{l}\text { 8. El estado de buena predisposición de los miembros de } \\
\text { la comunidad ciber-formativa }\end{array}$ & 86,1 & 8,3 & 0 & 0 & 5,6 \\
\hline 9. La organización curricular del programa & 69,4 & 22,2 & 2,8 & 0 & 5,6 \\
\hline 10. El clima generado en la comunidad ciber-formativa & 80,6 & 16,7 & 0 & 0 & 2,8 \\
\hline $\begin{array}{l}\text { 11. La adecuación de las propuestas de trabajo a tu } \\
\text { estilo de aprendizaje }\end{array}$ & 66,7 & 25 & 5,6 & 0 & 5,6 \\
\hline $\begin{array}{l}\text { 12. La posibilidad ofrecida de expresar resultados de } \\
\text { aprendizaje en formatos no escritos (audios, videos, } \\
\text { presentaciones, videoforo, etc.) }\end{array}$ & 77,8 & 16,7 & 2,8 & 0 & 2,8 \\
\hline $\begin{array}{l}\text { 13. El nivel de alfabetización digital conseguido durante } \\
\text { el programa formativo }\end{array}$ & 69,4 & 25 & 2,8 & 0 & 2,8 \\
\hline
\end{tabular}




\begin{tabular}{|c|c|c|c|c|c|}
\hline Ítem/ Porcentajes & $\begin{array}{l}\text { Mu- } \\
\text { cho }\end{array}$ & $\begin{array}{l}\text { Bas- } \\
\text { tante }\end{array}$ & Poco & $\begin{array}{l}\text { Muy } \\
\text { poco }\end{array}$ & $\mathrm{Ns} / \mathrm{Nc}$ \\
\hline $\begin{array}{l}\text { 14. Las relaciones ciber-personales con el equipo docen- } \\
\text { te surgidas en el desarrollo del programa formativo }\end{array}$ & 72,2 & 16,7 & 2,8 & 2,8 & 5,6 \\
\hline $\begin{array}{l}\text { 15. Las relaciones ciber-personales con el alumnado } \\
\text { surgidas en el desarrollo del programa formativo }\end{array}$ & 61,1 & 27,8 & 2,8 & 2,8 & 5,6 \\
\hline $\begin{array}{l}\text { 16. Los materiales de video artesanal elaborados por los } \\
\text { actores de la comunidad ciber-formativa }\end{array}$ & 77,8 & 8,3 & 5,6 & 2,8 & 5,6 \\
\hline $\begin{array}{l}\text { 17. La preocupación del profesorado por evitar estados } \\
\text { de ansiedad }\end{array}$ & 75 & 11,1 & 8,3 & 0 & 5,6 \\
\hline $\begin{array}{l}\text { 18. La preocupación del profesorado por evitar estados } \\
\text { de frustración }\end{array}$ & 80,6 & 11,1 & 5,6 & 0 & 2,8 \\
\hline $\begin{array}{l}\text { 19. La preocupación del profesorado por evitar estados } \\
\text { de desgana }\end{array}$ & 72,2 & 16,7 & 2,8 & 0 & 2,8 \\
\hline $\begin{array}{l}\text { 20. La preocupación del profesorado por conseguir } \\
\text { estados de disfrute }\end{array}$ & 80,6 & 13,9 & 2,8 & 0 & 2,8 \\
\hline 21. La realización de aprendizajes lúdicos & 77,8 & 11,1 & 5,6 & 0 & 5,6 \\
\hline 22. La preocupación por mantenerte activo/a & 80,6 & 16,7 & 2,8 & 0 & \\
\hline 23. El matiz afectivo existente en los procesos didácticos & 66,7 & 19,4 & 5,6 & 0 & 5,6 \\
\hline $\begin{array}{l}\text { 24. Las innovaciones tecnológico-didácticas ofrecidas en } \\
\text { el programa }\end{array}$ & 58,3 & 27,8 & 5,6 & 0 & 5,6 \\
\hline $\begin{array}{l}\text { 25. La posibilidad de aplicar lo positivamente vivido en } \\
\text { tu ámbito personal }\end{array}$ & 75 & 22,2 & 0 & 0 & 2,8 \\
\hline $\begin{array}{l}\text { 26. La posibilidad de aplicar lo positivamente vivido en } \\
\text { tu ámbito laboral }\end{array}$ & 69,4 & 13,9 & 2,8 & 2,8 & 11,1 \\
\hline 27. El sentirse acogido dentro de la experiencia formativa & 72,2 & 25 & 0 & 0 & 2,8 \\
\hline 28. El observar dinámicas de ayuda entre el alumnado & 66,7 & 27,8 & 2,8 & 0 & 2,8 \\
\hline $\begin{array}{l}\text { 29. El percibir dinámicas de colaboración entre el profe- } \\
\text { sorado }\end{array}$ & 66,7 & 22,2 & 0 & 0 & 11,11 \\
\hline 30. El haber recibido un trato respetuoso & 86,1 & 11,1 & 0 & 0 & 2,8 \\
\hline $\begin{array}{l}\text { 31. El haber percibido altas expectativas hacia el alum- } \\
\text { nado por parte del profesorado }\end{array}$ & 77,8 & 19,4 & 0 & 0 & 2,8 \\
\hline $\begin{array}{l}\text { 32. El haberse sentido igual de valorado que el resto de } \\
\text { compañeros/as }\end{array}$ & 80,6 & 16,7 & 0 & 0 & 2,8 \\
\hline 33. El buen nivel de accesibilidad de la plataforma & 72,2 & 16,7 & 0 & 2,8 & 8,3 \\
\hline 34. La facilidad de uso de la plataforma & 75 & 11,1 & 5,6 & 2,8 & 5,6 \\
\hline $\begin{array}{l}\text { 35. La incorporación de medidas que favorecen la aten- } \\
\text { ción a la diversidad }\end{array}$ & 83,3 & 8,3 & 0 & 0 & 8,3 \\
\hline $\begin{array}{l}\text { 36. La implicación del alumnado en su propio proceso } \\
\text { de aprendizaje }\end{array}$ & 77,8 & 19,4 & 0 & 0 & 2,8 \\
\hline $\begin{array}{l}\text { 37. La preocupación por apoyar el proceso de aprendiza- } \\
\text { je de todo el alumnado }\end{array}$ & 77,8 & 19,4 & 0 & 0 & 2,8 \\
\hline $\begin{array}{l}\text { 38. Aprovechar la riqueza de la diversidad de alumnado } \\
\text { y profesorado como un recurso para el aprendizaje }\end{array}$ & 83,3 & 13,9 & 2,8 & 0 & 0 \\
\hline
\end{tabular}


En lo referente al bienestar percibido por la cultura inclusiva generada, los resultados de los ítems número 14 y 15, referidos a las relaciones ciber-personales, ponen de manifiesto que prácticamente el $90 \%$ del alumnado se siente bastante o muy satisfecho con las relaciones establecidas con el equipo tutorial y con sus propios compañeros/as. En este mismo contexto, cabe destacar que prácticamente el 100\% de los respondientes está bastante o muy satisfecho con el trato respetuoso recibido (ítem 30).

Abundando en las razones de estas altas cotas de bienestar declaradas, los ítems 17, 18 y 19, indican que la evitación de episodios de ansiedad, de frustración y de desgana desde una adecuada acción tutorial afectiva e inclusiva, en la que el uso de la videoconferencia ha jugado un papel destacado (ítem 6 y 7), han sido decisivas para el mantenimiento del bienestar declarado (indicadores de evitación valorados como bastante o mucho por 9 de cada 10 respondientes). El ejercicio de la tutoría afectiva ha dado como resultado igualmente altas cotas de bienestar derivadas de la constante preocupación del profesorado por conseguir estados de disfrute durante el proceso formativo (ítem 20 y 23).

Tal ejercicio se ha caracterizado por el acompañamiento afectivo del profesorado desde una percepción de altas expectativas hacia el alumnado y desde un trato ecuánime respetando las diferencias individuales (ítem 5, 31 y 32). Las puntuaciones declaradas en estos indicadores ponen de manifiesto que prácticamente el $100 \%$ del alumnado sustancia su bienestar desde la generación de estas dinámicas. Este conjunto de indicadores ponen de manifiesto que el clima positivo creado por la comunidad ciber-educativa ha redundado decisivamente en el bienestar de sus miembros durante el desarrollo del diploma (ítem 10).

La praxis de esta cultura inclusiva se analiza igualmente en el cuestionario con la formulación de un conjunto de ítems que analizan la influencia de estrategias y recursos cuyo buen uso favorece igualmente la percepción generalizada de bienestar. La constatación de la importancia de aprovechar la riqueza de la diversidad como recurso de aprendizaje (ítem 38) y de incorporar medidas de atención a dicha diversidad (ítem 35), con una alta valoración otorgada por la práctica totalidad del alumnado de estos indicadores $(97,2 \%$ y $91,6 \%$, respectivamente), unido al ejercicio de la flexibilidad didáctica (ítem 1), de la atención a las dificultades personales (ítem 2) y a los estilos de aprendizaje (ítem 11), en un contexto de aprendizaje lúdicos (ítem 21) cuyas estimaciones son 
igualmente muy altas; son pruebas evidentes del éxito de los enfoques y buenas prácticas inclusivas realizadas desde la modalidad de blended learning.

Finalmente, cabe reseñar en esta selección de comentarios la constatación de que las innovaciones tecnológico-didácticas aplicadas (ítem 24), la expresividad de resultados en diversos formatos (ítem 12), la creación de vídeos artesanales (ítem 16) en un contexto curricular flexible (ítem 9), accesible (ítem 33) y usable (ítem 34); constituyen un conjunto de ítems que igualmente son altamente valorados por 9 de cada 10 participantes, lo que ha incidido sustancialmente al mantenimiento del clima de bienestar y de satisfacción generalizada que venimos subrayando.

\section{Discusión}

El análisis de resultados realizado sirve para argumentar sobre el grado de consecución del objetivo general de este trabajo. Los altos niveles de bienestar declarados confirman su relación directa con el favorable cumplimiento de las expectativas depositadas por el alumnado en el programa, con el adecuado ejercicio de la participación y del acompañamiento tutorial y con el desarrollo de actitudes y hábitos positivos para la construcción de aprendizajes.

La elección de la modalidad de blended learning ha facilitado la consecución de estas altas tasas de satisfacción y de predisposición de actitudes positivas hacia el aprendizaje, tal como proponen López-Pérez, Pérez-López \& Rodríguez-Ariza (2011). En esta experiencia, han resaltado especialmente el alto grado de compromiso asumido por el alumnado y la gran perseverancia demostrada, que, junto a otros factores, han incidido favorablemente en la consecución de una alta motivación y de un nivel de ejecución didáctica que se ha traducido en una bajísima tasa de abandono.

Tal como proponen en su investigación Hernández-Sánchez \& Ortega (2015, p.24), el ejercicio de la tutoría afectiva 'requiere de una serie de ajustes basados en el establecimiento de un elearning afectivo que incida en una acción tutorial impregnada de afectividad como elemento transversal a todas las funciones y decisiones docentes'. En esta investigación ha quedado explicitada la relación existente entre el modelo de tutoría afectiva implementado y su combinación con el liderazgo com- 
partido ejercido desde la coordinación del programa con el grado de bienestar conseguido y declarado por el alumnado participante.

La expectativa expresada inicialmente por la mayoría de los estudiantes de disfrutar aprendiendo durante el desarrollo del programa ha sido ampliamente conseguida gracias a la preocupación del equipo tutorial por facilitar espacios de disfrute, cuya energía positiva ha conducido a estados óptimos continuados de experiencia interna (flow). Estos flujos de optimismo y bienestar han permitido minimizar la aparición de situaciones indeseables de ansiedad, frustración y desgana en el alumnado participante, a la vez que le han ayudado a superar ciertas dificultades inicialmente expresadas tales como la falta de tiempo, el simultanear el estudio y el trabajo y la ausencia de experiencia de formación en entornos virtuales.

La cultura inclusiva generada, adoptando una visión holística que ha vertebrado aspectos emocionales, sociales, culturales y psicológicos (Patton, 2013), ha permitido que las buenas prácticas realizadas en el campus virtual y en las sesiones presenciales, aprovechen la riqueza de la diversidad como recurso de aprendizaje, promoviendo innumerables acciones de atención a dicha diversidad. Lo que unido a la flexibilidad didáctica y organizativa y al respecto de las peculiaridades del aprendizaje, ha favorecido el establecimiento de un clima positivo generador de bienestar en los miembros de la comunidad ciber-formativa. Siguiendo la visión de Moliner, Moliner \& Sales (2014, p. 285), se vislumbra la creación de una 'comunidad segura, acogedora, colaboradora y estimulante, en la que cada uno es valorado, lo cual es la base fundamental para que todo el alumnado obtenga mayores niveles de logro y éxito'.

Como sencilla aportación final a esta discusión se presenta, en forma gráfico, la arquitectura conceptual que sustenta el cuestionario en lo referente a los indicadores que analizan el bienestar generado en el programa formativo, cuya estimación ha sido el propósito central de esta investigación.

En la figura 4 se ha diseñado un mapa de indicadores para la evaluación continua que organiza las evidencias seleccionadas en el estudio en función de las dimensiones y subdimensiones recogidas en el Index para la inclusión, publicado por Booth \& Ainscow (2011). El hemisferio superior de la gráfica contiene las evidencias relacionadas con la cultura inclusiva desde la perspectiva de la construcción de la comunidad y el cultivo de valores inclusivos. El hemisferio inferior, por su parte, expresa 
la dimensión aplicada de esa cultura referida a la movilización de recursos didácticos y a la organización de las experiencias para la construcción de aprendizajes.

\begin{tabular}{|c|c|c|}
\hline $\begin{array}{l}\text { relaciones ciber-personales con el equipo docente } \\
\text { gidas en el desarrollo del programa formativo. } \\
\text { relaciones ciber-personales con el alumnado surgidas } \\
\text { l desarrollo del programa formativo. }\end{array}$ & \multicolumn{2}{|c|}{$\begin{array}{l}\text { comunidad ciber-formativa. } \\
\text { El clima generado en la comunidad ciber-formativa. } \\
\text { El interés del profesorado por evitar estados de ansiedad. } \\
\text { El interés del profesorado por evitar estados de frustración. } \\
\text { El interés del profesorado por evitar estados de desgana. } \\
\text { El interés del profesorado por conseguir estados de disfrute. } \\
\text { El haber percibido altas expectativas hacia el alumnado por } \\
\text { parte del profesorado. }\end{array}$} \\
\hline $\begin{array}{l}\text { Construir } \\
\text { comunidad }\end{array}$ & $\begin{array}{l}\text { Valores } \\
\text { inclusivos }\end{array}$ & $\begin{array}{l}\text { El haberse sentido igual de valorado que el resto } \\
\text { de compañeros/as. } \\
\text { La preocupación por apoyar el proceso de } \\
\text { aprendizaje de todo el alumnado. }\end{array}$ \\
\hline $\begin{array}{l}\text { Movilización } \\
\text { de recursos }\end{array}$ & $\begin{array}{l}\text { Organización } \\
\text { del } \\
\text { aprendizaje }\end{array}$ & $\begin{array}{l}\text { La flexibilidad ofrecida en ciertos plazos. } \\
\text { El hecho de que se hayan tenido en cuenta } \\
\text { ciertas eventualidades y dificultades } \\
\text { personales. }\end{array}$ \\
\hline $\begin{array}{l}\text { El nivel de alfabetización digital conseguido durante el } \\
\text { programa formativo. } \\
\text { Los materiales de video artesanal elaborados por los } \\
\text { actores de la comunidad ciber-formativa. } \\
\text { La realización de aprendizajes lúdicos. } \\
\text { Las innovaciones tecnológico-didácticas ofrecidas en el } \\
\text { programa. } \\
\text { La posibilidad de aplicar lo positivamente vivido en tu } \\
\text { ámbito personal. } \\
\text { La posibilidad de aplicar lo positivamente vivido en tu } \\
\text { ámbito laboral. } \\
\text { La incorporación de medidas que favorecen la atención a } \\
\text { la diversidad. } \\
\text { Aprovechar la riqueza de la diversidad de alumnado y } \\
\text { profesorado como un recurso para el aprendizaje. }\end{array}$ & \multicolumn{2}{|c|}{$\begin{array}{l}\text { La colaboración del alumnado en la co-evaluación realizada al } \\
\text { comentar tareas desde espacios colaborativos (espacios de } \\
\text { foro). } \\
\text { La claridad de criterios de evaluación expresada en las } \\
\text { rúbricas. } \\
\text { La celebración de videoconferencias grupales. } \\
\text { La celebración de videoconferencias individuales. } \\
\text { La organización curricular del programa. } \\
\text { La adecuación de las propuestas de trabajo a tu estilo de } \\
\text { aprendizaje } \\
\text { La posibilidad ofrecida de expresar resultados de aprendizaje en } \\
\text { formatos no escritos (audios, videos, presentaciones, videoforo } \\
\text { etc.). } \\
\text { El interés por mantenerte activo/a. } \\
\text { El buen nivel de accesibilidad de la plataforma. } \\
\text { La facilidad de uso de la plataforma. } \\
\text { La implicación del alumnado en su propio proceso } \\
\text { de aprendizaje. }\end{array}$} \\
\hline
\end{tabular}

Figura 4. Mapa de indicadores para la evaluación continua de un programa de blended learning inclusivo (elaboración propia).

A modo de epílogo puede señalarse que la inclusión de parámetros de evaluación del bienestar generado en procesos formativos de blended learning inclusivo es una práctica que comienza a aplicarse en aquellas instituciones y universidades que trabajamos por una educación a distancia en entornos virtuales humanizadores y afectivos, en los que se valoran tanto las componentes intelectivas como las emocionales.

Los resultados obtenidos en esta experiencia educativa indican que la comunidad ciber-formativa en la que se ha desarrollado el diploma no solo está movida por ideales de justicia, equidad y solidaridad inclusiva, sino que además ha conseguido vibrar fuertemente con las emociones positivas derivadas del alto bienestar generado. 


\section{Referencias bibliográficas}

Ainscow, M. (2009). Developing inclusive education systems: What are the levers for change? In P. Hick \& G. Thomas (Coords.), Inclusion and diversity in education (volume 2) (pp. 1-13). London: SAGE.

Booth, T. \& Ainscow. A. (2011). Index for inclusion: developing learning and participation in schools. Bristol: Centre for Studies on Inclusive Education.

Cabero, J., Llorente, M.C. \& Puente, A. (2010). La satisfacción de los estudiantes en red en la formación semipresencial. Comunicar, 35 (XVIII), 149-157. Recuperado de http://dx.doi.org/10.3916/C35-2010-03-08

Carr, A. (2007). Psicología positiva. La ciencia de la felicidad. Barcelona: Paidós.

Colás, M.P., Buendía, L. \& Hernández, F. (2009). Competencias científicas para la realización de una tesis doctoral. Barcelona: REDES.

Collopy, R. \& Marshall, J. (2009). To Blend or Not To Blend: Online and Blended Learning Environments in Undergraduate Teacher Education. Issues in teacher education, 2 (XVIII), 285-101. Recuperado de: http://goo.gl/k1jfsl

Csikszentmihalyi, M. (2013). Fluir (flow). Una psicología de la felicidad. Barcelona: Kairós.

Durán, D. \& Giné, C. (2011). La formación del profesorado para la educación inclusiva: Un proceso de desarrollo profesional y de mejora de los centros para atender la diversidad. Revista Latinoamericana de Educación Inclusiva, 2 (V), 153-170. Recuperado de http://goo.gl/qudymc

Echeita, G. \& Ainscow, M. (2011). La educación inclusiva como derecho. Marco de referencia y pautas de acción para el desarrollo de una revolución pendiente. Tejuelo, 12 (IV), 26-46. Recuperado de: http://goo.gl/f9o4dF

Garrison, D. R. (2011). E-Learing in the 21 th century. A framework for research and practice. New York: Routledge.

Garrison, D.R. \& Vaughan, N.D. (2008). Blended learning in higher education. San Francisco: Jossey-Bass.

Hernández-Sánchez, A.M. \& Ortega, J.A. (2015). Aprendizaje electrónico afectivo: un modelo innovador para desarrollar una acción tutorial virtual de naturaleza inclusiva. Formación Universitaria, 2 (VIII), 19-26. DOI: 10.4067/S0718-50062015000200004

Llorente, M.C. \& Cabero, J. (2009). La formación semipresencial a través de redes telemáticas (blended learning). Barcelona: Da Vinci.

Llorente, M.C., Cabero, J. \& Barroso, J. (2015). El papel del profesor y el alumnado en los nuevos entornos tecnológicos. In J. Cabero \& J. Barroso, Nuevos retos en tecnología educativa. Madrid: Síntesis.

López-Pérez, M.V., Pérez-López, M.C. \& Rodríguez-Ariza, L. (2011). Blended learning in higher education: Students' perceptions and their relation to outcomes. Computer and education, 56, 818-826. DOI: 10.1016/j.compedu.2010.10.023

Marín, V., Gutiérrez, P. \& Aguaded, J.I. (2014). La competencia digital en educación inclusiva. In V. Marín, Desarrollando la competencia digital desde la educación inclusiva. Barcelona: Da Vinci.

Martínez, A. \& Gómez, J.L. (2013). Escuelas inclusivas singulares. Madrid: Ciclo Grupo 5. 
Moliner, L., Moliner, O. \& Sales, A. (2014). Conocer la cultura de un centro y las miradas del profesorado sobre la diversidad sociocultural y personal de la comunidad como punto de arranque para la transformación educativa. Cultura y Educación, 3 (XXII), 283-296. DOI: http://dx.doi.org/10.1174/113564010804932193

Organización de las Naciones Unidas (2006). Convención sobre los derechos de las personas con discapacidad. Recuperado de http://goo.gl/ANA1fc

Patton, M.Q. (2013). The future of evaluation in society. Top ten trends plus one. In S.I. Donaldson (Ed.). The future of evaluation in society. A tribute to Michael Scriven (pp. 45-62). USA: Information Age Publishing.

Pérez, A.I. (2012). Educarse en la era digital. Madrid: Morata.

Sala, I., Sánchez, S., Giné, C. \& Díaz, E. (2014). Análisis de los distintos enfoques del paradigma del diseño universal aplicado a la educación. Revista latinoamericana de educación inclusiva, 1 (VIII), 143-152. Recuperado de http://goo.gl/8mrFso

UNESCO (2009). Directrices sobre políticas de inclusión en la educación. París: UNESCO. Recuperado de http://goo.gl/S7MisH 
